\title{
Uma Estratégia de Redução do Estresse entre Estudantes Médicos
}

\section{A Strategy for Stress Reduction among Medical Students}

\section{PALAVRAS-CHAVE}

- Meditação.

- Estresse.

- Estudantes de Medicina.

- Educação Médica.

- Medicina Integrativa.
Fernanda Martin Catarucci $i^{I}$ D Talita Cardoso Rossi ${ }^{I} \mathbb{D}$

Vânia Hercília Talarico Bruno ${ }^{I}$ (D) Ivan da Silva Beteto ${ }^{I}$ (D) Pedro Henrique Leonetti Habimorad ${ }^{I}$ (iD Madeline Susan Andrews ${ }^{I I}($ D Emmanuel A. Burdmann ${ }^{I I I}$ Karina Pavão Patrício ${ }^{I}$ (D)

\section{RESUMO}

Introdução: $O$ curso de graduação em Medicina expõe os alunos a uma quantidade significativa de estresse, o que pode gerar consequências negativas para o aprendizado, a motivação e o contato com os pacientes. Algumas técnicas e práticas têm sido indicadas para auxiliar no manejo e na redução do estresse, como é o caso da meditação que já é utilizada em escolas médicas. Este estudo avaliou os efeitos de um programa de redução do estresse e desenvolvimento da empatia em medicina (Redemed ${ }^{\odot}$ ) sobre a percepção de estresse de seus participantes e possíveis grupos de acolhimento. Método: Este é um ensaio quase experimental cuja amostra foi composta por 40 estudantes que compuseram o grupo controle e 47 alunos de um grupo de intervenção que participaram de oito encontros semanais de duas horas divididas em: 30 minutos de teoria sobre como o estresse influencia o estado de saúde, 60 minutos de vivências interpessoais e 30 minutos de ioga e meditação. Ambos os grupos, antes e depois do curso, responderam ao questionário sobre estresse percebido (PSS - Escala de Cohen). Resultados: Após os oito encontros semanais, o grupo intervenção apresentou melhora significativa $(p=0,030)$, demonstrando que a participação no curso Redemed ${ }^{\odot}$ mostrou-se eficaz no controle do estresse entre os estudantes do presente estudo. Os alunos também foram questionados quanto à autopercepção sobre se sentirem ou não apoiados por outros grupos. Os três grupos de acolhimentos mais citados entre os alunos, tanto do grupo ativo como do controle, foram: amigos/família, centro acadêmico e a equipe do esporte que praticavam. Após as oito semanas, enquanto o grupo controle permaneceu com as mesmas indicações, no grupo intervenção foram citados: amigos/família, Redemed ${ }^{\odot}$ e centro acadêmico. Conclusão: Este estudo mostrou que o programa Redemed ${ }^{\odot}$, com encontros durante oito semanas utilizando a meditação como sua técnica central, foi eficaz na redução do estresse percebido pelos estudantes de Medicina que participaram dessa intervenção quando comparado ao grupo controle $(p=0,000)$. As práticas integrativas e complementares podem ser uma ferramenta importante dentro das escolas médicas, de modo a levar os estudantes a lidar melhor com o estresse a que estão expostos ao longo do curso.

\footnotetext{
${ }^{\mathrm{I}}$ Universidade Estadual Paulista Júlio de Mesquita Filho, Botucatu, São Paulo, Brasil.

IIInstituto Visão Futuro, São Paulo, São Paulo, Brasil.

${ }^{\mathrm{II}}$ Universidade de São Paulo, São Paulo, São Paulo, Brasil.
} 


\section{KEYWORDS}

- Meditation.

- Stress.

- Medical Students.

- Medical Education.

- Integrative Medicine.

\section{ABSTRACT}

Introduction: Undergraduate medical students experience a considerable amount of stress, which can negatively affect their learning, motivation and contact with patients. Some techniques and practices for stress management and reduction, such as meditation, have been recommended and used in medical schools. This study evaluated the effects of a Stress Reduction and Empathy Development Program in Medicine $\left(R E D E M E D^{\odot}\right)$ on participants' perception of stress and possible support groups. Method: This is a quasiexperimental trial whose sample comprised 40 students in a control group and 47 students in an intervention group. The students in the intervention group participated in eight weekly sessions lasting two hours each. The course hours were divided into: 30 minutes of theoretical content on how stress influences one's health, 60 minutes of interpersonal practices and 30 minutes of yoga and meditation practices. Both groups, before and after the program, answered the questionnaire on perceived stress (PSS - Cohen's scale). Results: After the eight weekly meetings, the intervention group showed significant improvement $(p=0.030)$, showing that participation in the REDEMED ${ }^{\oplus}$ course proved to be effective in stress control among the students in the study. The students were also questioned about their self-perception of whether or not they felt they were supported by any other groups. The three support groups most often referred to by the students, in both the intervention and the control group, were: friends/family, the Students' Union and their sports team. After eight weeks, while the control group still referred to the same groups, the intervention group mentioned friends/family, REDEMED ${ }^{\odot}$ and the Students' Union. Conclusion: This study showed that the REDEMED ${ }^{\odot}$ program, meeting for eight weeks using meditation as its central technique, was effective in reducing the stress perceived by medical students who participated in this intervention when compared to the control group $(p=0,000)$. Integrative and complementary practices can be an important tool within medical schools, empowering students to better cope with the stress they are exposed to throughout the course.

Recebido em 13/5/20

Aceito em 21/6/20

\section{INTRODUÇÃO}

Pesquisas demonstram que $12 \%$ a $18 \%$ dos estudantes universitários apresentam algum tipo de transtorno mental diagnosticável ${ }^{1}$. Entre as enfermidades, estão o estresse, a depressão e os sintomas ansiosos que são mais acentuados nos estudantes de Medicina ${ }^{2-5}$. Essa particularidade dentro da Medicina pode ser explicada por alguns fatores: cobrança no desempenho acadêmico, responsabilidades clínicas, desenvolvimento de uma identidade profissional, exposição à morte e ao sofrimento humano, conflitos étnicos e cobrança excessiva do paciente, de seus familiares e dos próprios profissionais ${ }^{6}$. Além desses fatores, alguns estudos vêm considerando que parte do estresse dessa população pode ser proveniente do esforço associado a metas futuras e à escassez de tempo para dedicar à vida pessoal. Considera-se que, durante o curso (de período integral), há uma demasiada preocupação relacionada à entrada no mercado de trabalho ou ao ingresso em programas de residência médica, o que implica dedicação, sacrifício e resistência física e emocional dos alunos, resultando em pouco tempo para outras atividades ${ }^{1,6}$.

Em uma revisão da literatura sobre estresse, burnout e estratégias de enfrentamento em estudantes de Medicina pré-clínica, composta por 23 artigos, Fares et al. ${ }^{7}$ encontraram dados que variam de $20 \%$ (Nepal) a 90\% (Paquistão) para prevalência de estresse entre os estudantes. Já em um estudo multicêntrico, envolvendo 22 escolas médicas brasileiras (13 públicas e nove privadas) e com o objetivo de avaliar a qualidade de vida, habilidades emocionais e ambiente educacional de estudantes e residentes de saúde, investigou 1.350 indivíduos e mostrou que a prevalência de sintomas de depressão entre os estudantes de Medicina brasileiros (41,3\%) é superior à prevalência global relatada $(28 \%)^{5}$.
Lemos et al. ${ }^{8}$ concluíram que o estresse é uma das três razões mais citadas para justificar o uso de substâncias psicoativas entre estudantes de Medicina, segundo estudo realizado com 404 estudantes do primeiro ao sexto ano das duas maiores escolas de Medicina, uma pública e uma particular, em Salvador. Em relação ao uso de álcool e drogas, um estudo com 5.227 estudantes de graduação em Medicina do estado de São Paulo, no período de 1994 a 1995, mostrou um uso crescente de todas as drogas do primeiro ao sexto ano, principalmente de benzodiazepínicos, o que pode estar relacionado ao estresse causado pelo exame de residência a ser realizado e à sobrecarga de turnos, segundo os autores".

Nesse contexto de implicações para a saúde mental dos estudantes e a qualidade de sua prática profissional, algumas propostas de intervenção na educação médica foram feitas para minimizar esse sofrimento, sendo a prática de mindfulness uma delas. Dobkin e Hutchinson ${ }^{10}$ conduziram uma revisão dos programas de mindfluness com práticas de meditação implementadas em várias faculdades de Medicina de universidades dos Estados Unidos (Rochester, Brown, Drexel, Duke, Georgetown, Lowas, Jefferson, Massachusetts), do Canadá (Toronto, Montreal, McGill) e da Austrália (Monash). Os resultados mostraram a eficácia de tais práticas na redução de emoções negativas e estresse, bem como no aumento da atenção, empatia e compaixão.

A meditação pode ser definida como uma prática autorregulatória da atenção, compreendendo técnicas que causam relaxamento mental e muscular. Trata-se de um estado que é exclusivamente autoinduzido com base no foco ${ }^{11}$. Associadas ao conhecimento originado das filosofias orientais, como a ioga e o budismo, essas técnicas fazem parte de formas tradicionais de conhecimento e sistemas médicos que consideram uma

REVISTA BRASILEIRA DE EDUCAÇÃO MÉDICA

2 44 (3) : e104; 2020 
visão integral do ser humano ${ }^{12-14}$. Desde a década de 1970 , as pesquisas sobre meditação tornaram-se mais intensas, e agora essa prática pode ser recomendada como terapia integrativa para aliviar os sintomas de estresse e depressão, entre outros ${ }^{15}$.

Em uma revisão da literatura, Shapiro et al. ${ }^{16}$ descobriram que técnicas de enfrentamento do estresse, como meditação e promoção de grupos de apoio à expressão emocional para estudantes de Medicina, resultaram em aumento da função imunológica, diminuição dos sintomas de depressão e ansiedade, aumento da empatia e capacidade de resolver conflitos e lidar com o estresse.

Assim, este estudo investigou se um programa de oito semanas sobre gerenciamento do estresse e desenvolvimento da empatia poderia interferir na percepção do estresse em estudantes de Medicina.

\section{MÉTODOS}

Um estudo prospectivo, controlado e quase experimental foi realizado para avaliar os efeitos do Programa de Redução de Estresse e Desenvolvimento da Empatia em Medicina $\left(\right.$ Redemed $^{\odot}$ ) na percepção do estresse por estudantes de Medicina. O programa foi desenvolvido e patenteado por Andrews ${ }^{17}$.

O projeto de pesquisa foi aprovado pelo Comitê de Ética em Pesquisa da Faculdade de Medicina de Botucatu da Universidade Estadual Paulista "Júlio de Mesquita Filho" (FMB/Unesp), com Parecer no 1.068 .260 emitido em 19 de maio de 2015, e recebeu o financiamento da Fundação de Amparo à Pesquisa do Estado de São Paulo - Fapesp (Processo ${ }^{\circ}$ 2015/10854-2).

Inicialmente, todos os alunos do curso de Medicina da FMB, do primeiro ao sexto ano da graduação, foram convidados para assistir a uma palestra, na qual se apresentou a proposta da pesquisa e os discentes puderam esclarecer suas dúvidas.

Os alunos que tiveram interesse em participar da pesquisa receberam o Termo de Consentimento Livre e Esclarecido (TCLE) e compuseram o grupo intervenção, que realizou o curso Redemed ${ }^{\circ}$ oferecido em oito encontros semanais de duas horas presenciais. O grupo controle foi composto por graduandos de Medicina que assistiram à palestra, mas, por incompatibilidade de horário, não puderam participar do Redemed $^{\odot}$. Também fizeram parte do grupo controle discentes indicados pelos integrantes do grupo ativo, que convidaram colegas de diferentes anos que se dispuseram a colaborar com o estudo. Esses alunos também preencheram o TCLE antes de realizarem as avaliações quantitativas. A coleta de dados ocorreu no ano de 2016.

O programa Redemed ${ }^{\circ}$, ministrado pela Dra. Susan Andrews, foi constituído de uma parte teórica e uma parte prática. Na parte teórica, abordaram-se as pesquisas que correlacionam os benefícios da meditação e respiração no sistema nervoso central e seus efeitos sobre o estresse diário vivenciado pelos alunos e profissionais da área da saúde em geral. $\mathrm{Na}$ parte prática, trabalharam-se exercícios para o gerenciamento do estresse, com a utilização de ferramentas como o psicodrama, em que os próprios alunos representaram cenas do cotidiano no contexto clínico, além de dinâmicas em grupo e em duplas para o exercício da escuta, fala e postura empáticas, produção de ilustrações e preenchimento de questionários de autoavaliação do comportamento.

O curso semanal de duas horas foi assim dividido: 30 minutos de teoria sobre como o estresse influencia o estado de saúde, a importância da empatia, como isso provoca outros problemas na vida pessoal e profissional dos futuros médicos; 60 minutos de práticas interpessoais; e, por fim, 30 minutos de ioga e meditação.

Ambos os grupos preencheram a ficha do perfil elaborada pela pesquisadora e responderam ao questionário de estresse percebido (Perceived Stress Scale - PSS) de Cohen antes e depois do curso Redemed ${ }^{\odot}$. Esse questionário respondido individualmente em sala separada, antes da coleta de sangue.

O questionário de Cohen, já validado em portuguêes ${ }^{18,19}$, tem 14 questões com opções de resposta que variam de 0 a $4(0=$ nunca; 1 = quase nunca; $2=$ às vezes; 3 = quase sempre $4=$ sempre). As questões com conotação positiva $(4,5,6,7,9,10$ e 13$)$ têm sua pontuação somada invertida, da seguinte maneira, $0=4,1=3,2=2,3=1$ e $4=0$. As demais questões são negativas e devem ser somadas diretamente. $\mathrm{O}$ total da escala é a soma das pontuações das 14 questões, e os escores podem variar de 0 a 56.

A PSS é uma escala geral que pode ser usada em diversos grupos etários, desde adolescentes até idosos, pois não contém questões específicas do contexto. A ausência de questões específicas de contexto é um fator importante na escala e, provavelmente, a razão pela qual essa escala foi validada em diversas culturas ${ }^{20-22}$.

A PSS permite estudos com uma perspectiva epidemiológica, pois demanda pouco tempo para o seu preenchimento e apresenta características psicométricas satisfatórias. Além disso, esse instrumento avalia o estresse em um contexto global, característica importante na comparação de dados de pesquisa ${ }^{18}$.

Para a análise estatística, utilizou-se o software Statistical Package for the Social Science for Windows (SPSS), versão 21.0. A comparação entre os grupos em relação aos potenciais confundidores foi feita pelos testes qui-quadrado e exato de Fisher e Mann-Whitney, seguidos da comparação entre grupos em relação às evoluções por Mann-Whitney. Em seguida, ajustou-se um modelo de regressão linear normal múltipla para estimar o efeito da intervenção sobre a evolução da pontuação do estresse, depois de corrigido o efeito confundidor do sexo. As diferenças foram consideradas estatisticamente significativas se $\mathrm{p}<0,05$.

Após a palestra, 73 alunos se inscreveram no programa Redemed ${ }^{\circ}$ e foram incluídos no grupo intervenção, e 59 estudantes de Medicina indicados pelos integrantes do grupo intervenção formaram o grupo controle. No grupo intervenção, houve um abandono de $25 \%$ dos alunos durante o curso, e o número de participantes diminuiu de 73 para 55 alunos. A principal razão apontada pelos alunos que abandonaram o programa foi a incompatibilidade de tempo e a sobrecarga de atividades da graduação.

No grupo controle, houve uma perda de cinco estudantes que não retornaram para a segunda avaliação. Assim, a amostra incluiu 109 casos, sendo 55 no grupo intervenção e 54 no grupo controle. Na análise dos dados, excluíram-se os questionários que foram preenchidos incorretamente e dificultaram a soma total; portanto, o estudo totalizou 47 estudantes no grupo intervenção e 40 no grupo controle. Os alunos preencheram os questionários individualmente. Uma análise da conclusão da pesquisa no momento da entrega da entrevista poderia minimizar a perda do número de sujeitos da pesquisa.

\section{RESULTADOS}

A amostra final foi composta por 87 alunos, sendo 47 no grupo intervenção e 40 no grupo controle, com predominância do sexo feminino

REVISTA BRASILEIRA DE EDUCAÇÃO MÉDICA

3 44(3) : e104; 2020 
(64\% do grupo intervenção e 42,5\% do controle), o que mostrou diferença estatisticamente significante $(\mathrm{p}=0,046)$.

Um modelo de regressão linear normal múltipla para estimar o efeito da intervenção sobre a evolução da pontuação do estresse foi realizado para corrigir o efeito confundidor do sexo. Nos grupos, apesar da diferença entre os sexos, esse resultado não impactou a intervenção na evolução do estresse (Tabela 2).

Na Tabela 1, também é possível observar que a média de idade foi de 22 anos tanto no grupo intervenção (de 18 a 32) como no grupo controle (de 18 a 28). Não se verificou diferença estatística $(p=0,673)$ e não houve diferença no número de participantes em cada ano do curso de graduação em Medicina ( $\mathrm{p}=0,385)$.

A maioria dos estudantes dos grupos intervenção (98\%) e controle (100\%) não praticava meditação no momento em que o curso Redemed ${ }^{\circ}$ foi realizado. Alguns estudantes de graduação nos grupos intervenção (34\%) e controle (20\%) relataram ter meditado em determinadas ocasiões antes do estudo.

Havia poucos que faziam uso de tabaco nos dois grupos (6,5\% no grupo intervenção e 7,5\% no grupo controle), mas muitos estudantes consumiam bebidas alcoólicas $(59,6 \%$ no grupo intervenção e $60 \%$ no grupo controle), o que mostrou que os grupos foram homogêneos em relação a esses hábitos ( $\mathrm{p}=1,00$ e $\mathrm{p}=0,96$, respectivamente).

Os alunos também foram questionados sobre a percepção de sentirem-se pertencentes aos outros grupos ou apoiados por eles. Embora a diferença não tenha sido significativa após o curso $(\mathrm{p}=0,069)$, o grupo intervenção apresentou um aumento (36\% para 59,6\%) do sentimento de apoio em relação ao grupo controle, que diminuiu (42,5\% para $40 \%$ ).

Os três grupos de apoio mencionados com mais frequência pelos alunos, tanto no grupo intervenção quanto no controle, foram: amigos/família, centro acadêmico e sua equipe esportiva. Após as oito semanas, enquanto o grupo controle ainda citava os mesmos grupos, o grupo intervenção fez referência aos amigos/à família, ao Redemed $^{\odot}$ e ao centro acadêmico.

Inicialmente, a aplicação do questionário PPS mostrou que, apesar de o grupo intervenção apresentar piores resultados em relação à percepção do estresse (32) do que o grupo controle $(27,5)$, essa diferença não foi estatisticamente significante $(\mathrm{p}=0,084)$ entre os estudantes antes do curso Redemed $^{\circ}$ (Tabela 3).

Entretanto, após os oito encontros semanais, o grupo intervenção apresentou melhora significativa $(\mathrm{p}=0,030)$ por conta da redução na soma da pontuação fornecida pelo instrumento aplicado (24), enquanto os resultados do grupo controle permaneceram praticamente inalterados $(27,5)$, como pode ser observado na Tabela 3.

No grupo intervenção, as questões que apresentaram as melhores diferenças em suas pontuações foram aquelas relacionadas a se sentir estressado (de 146 para 107), confiança para resolver os problemas pessoais (de 122 para 155) e percepção de que não conseguia lidar com todas as coisas que tinha que fazer (de 136 para 96).

\section{DISCUSSÃO}

De acordo com os resultados deste estudo, o grupo intervenção apresentou melhora significativa $(p=0,030)$ na percepção do estresse, avaliada pela aplicação do questionário PSS, o que corrobora a eficácia do curso Redemed $^{\odot}$ no gerenciamento do estresse entre os alunos da presente pesquisa. Considerando a alta prevalência de estresse em estudantes de
Medicina, diferentes tipos de intervenção foram desenvolvidos com o objetivo de auxiliar futuros profissionais na sua gestão, mas a literatura ainda apresenta limitações no campo metodológico, como a falta de grupos controle, estudos randomizados e o acompanhamento em longo prazo dos sujeitos submetidos à intervenção ${ }^{23}$. No entanto, resultados

\begin{tabular}{|c|c|c|c|}
\hline \multicolumn{4}{|c|}{$\begin{array}{c}\text { Tabela } 1 \\
\text { Perfil dos grupos controle e intervenção }\end{array}$} \\
\hline \multirow[b]{2}{*}{ Variáveis } & \multicolumn{2}{|c|}{ Grupo } & \multirow[b]{2}{*}{$p$} \\
\hline & $\begin{array}{l}\text { Controle } \\
(\mathrm{n}=40)\end{array}$ & $\begin{array}{l}\text { Intervenção } \\
\quad(\mathrm{n}=47)\end{array}$ & \\
\hline Idade (anos) & $22(18-28)$ & $22(18-32)$ & 0,673 \\
\hline Sexo masculino (\%) & $23(57,5 \%)$ & $17(36,2 \%)$ & 0,046 \\
\hline Ano da graduação & $2(1-6)$ & $3(1-6)$ & 0,385 \\
\hline $\begin{array}{l}\text { Número de alunos que meditavam } \\
\text { no momento }\end{array}$ & $0(0,0 \%)$ & $1(2,1 \%)$ & 1,000 \\
\hline $\begin{array}{l}\text { Número de alunos que já haviam } \\
\text { meditado em algum momento } \\
\text { antes do estudo }\end{array}$ & $8(20,0 \%)$ & $16(34,0 \%)$ & 0,144 \\
\hline Sensação de acolhimento inicial & $17(42,5 \%)$ & $17(36,2 \%)$ & 0,541 \\
\hline Sensação de acolhimento final & $16(40,0 \%)$ & $28(59,6 \%)$ & 0,068 \\
\hline $\begin{array}{l}\text { Número de alunos que fumavam } \\
\text { no momento }\end{array}$ & $3(7,5 \%)$ & $3(6,4 \%)$ & 1,000 \\
\hline $\begin{array}{l}\text { Número de alunos que bebiam no } \\
\text { momento }\end{array}$ & $24(60,0 \%)$ & $28(59,6 \%)$ & 0,967 \\
\hline
\end{tabular}

Fonte: Elaborada pelos autores.

\begin{tabular}{|c|c|c|c|c|c|}
\hline \multicolumn{6}{|c|}{$\begin{array}{l}\text { Regressão linear para estimar o impacto da intervenção sobre a } \\
\text { evolução do estresse depois de corrigido o efeito do sexo }\end{array}$} \\
\hline Variável & $\beta$ & ep & IC & & $\mathbf{p}$ \\
\hline Constante & 6,39 & 9,83 & $-13,16$ & 25,93 & 0,518 \\
\hline Intervenção & $-30,24$ & 10,68 & $-51,48$ & $-8,99$ & 0,006 \\
\hline Sexo masculino & 5,31 & 10,68 & $-15,94$ & 26,56 & 0,620 \\
\hline
\end{tabular}

Fonte: Elaborada pelos autores.

\begin{tabular}{|c|c|c|c|}
\hline \multicolumn{4}{|c|}{$\begin{array}{l}\text { Médias e níveis de significância }(\mathrm{p}<0,05) \text { quando se compararam } \\
\text { os resultados da PSS nos grupos controle e intervenção, antes e } \\
\text { depois do curso, Redemed }{ }^{\odot}, 2016\end{array}$} \\
\hline Variável & $\begin{array}{l}\text { Controle } \\
(\mathrm{n}=40)\end{array}$ & $\begin{array}{l}\text { Intervenção } \\
\qquad(n=47)\end{array}$ & $p$ \\
\hline Pontuação inicial do estresse & $27,5(07 / 51)$ & $32(09 / 46)$ & 0,084 \\
\hline Pontuação final do estresse & $27,5(09 / 49)$ & $24(05 / 42)$ & 0,030 \\
\hline Variação do estrese & $-3,4(-47 /-385)$ & $-29,4(-58 /-22)$ & 0,000 \\
\hline
\end{tabular}

Fonte: Elaborada pelos autores. 
positivos no gerenciamento do estresse, em que se utilizaram intervenções semelhantes à adotada nesta pesquisa, foram apontados em outros estudos com estudantes de Medicina e demais profissionais de saúde $e^{24-26}$.

A metodologia utilizada no Redemed $^{\odot}$ e os resultados encontrados neste estudo estão alinhados com uma revisão de literatura e metanálises conduzidas por Regehr et $\mathrm{al.}^{24}$, que concluíram que intervenções cognitivo-comportamentais, incluindo relaxamento e meditação, e técnicas baseadas na atenção plena são eficazes na redução dos sintomas de ansiedade em médicos e estudantes de Medicina. Essa revisão considerou um banco de dados contendo 1.992 artigos disponíveis até a data da pesquisa (fevereiro de 2013) e baseou-se em 87 títulos e resumos potencialmente interessantes; no entanto, a maioria deles não avaliou uma intervenção, mas aspectos relacionados à satisfação do paciente e ao apoio aos profissionais. Dos estudos que avaliaram a intervenção (técnicas cognitivas e comportamentais ou técnicas de atenção plena), selecionamse 12 que atendiam aos critérios dos revisores e compreendiam um total de 1.034 pessoas. Cinco desses estudos utilizaram períodos de intervenção variando de sete a 16 semanas, com reuniões semanais guiadas por um profissional experiente (como proposto pelo Redemed $^{\odot}$ ), e três deles aplicaram a PSS usada nesta pesquisa.

A revisão publicada por McConville et al. ${ }^{25}$ sobre os efeitos do treinamento de mindfulness no bem-estar psicológico, no aprendizado e no desempenho clínico dos estudantes de saúde em geral, que incluiu 19 estudos e um total de 1.815 participantes, também confirmou que esse tipo de intervenção reduz o estresse, a ansiedade e a depressão, e melhora o humor, a autoeficácia e a empatia dos alunos.

Van Dijk et al. ${ }^{26}$ realizaram um ensaio clínico controlado com 167 estudantes de Medicina da Duke University, de fevereiro de 2011 a maio de 2014, durante seu primeiro ano de estágio. Observaram-se benefícios positivos, como redução do estresse psicológico e de disfunções cognitivas, além de um aumento moderado da saúde mental e da satisfação com a vida durante os 20 meses de acompanhamento dos estudantes $(n=83)$ que foram alocados ao grupo participante em um treinamento baseado em mindfulness para redução do estresse (Mindfulness-Based Stress Reduction - MBSR). O MBSR consiste em um treinamento em grupo de oito semanas que visa reduzir o estresse e aumentar a atitude mental positiva, ensinando os participantes a estar alertas às sensações corporais, aos pensamentos e às emoções em uma atitude sem julgamento.

Uma intervenção de oito semanas envolvendo mindfulness associada à ioga e música suave com a equipe de uma unidade de terapia intensiva mostrou que os participantes reduziram a reatividade ao estresse no trabalho, melhorando seus marcadores psicológicos e biológicos em comparação aos do grupo controle ${ }^{27}$.

Moreira, Vasconcellos e Heath ${ }^{28}$ avaliaram as causas do estresse entre estudantes de Medicina e seus métodos de enfrentamento em um estudo com estudantes de uma faculdade de medicina em Montreal, no Canadá, e mostraram que a racionalidade científica que caracteriza o treinamento médico não valoriza os aspectos subjetivos da vida cotidiana dos estudantes. Portanto, os estudantes não encontram abertura para compartilhar sentimentos, ansiedade e preocupações no contexto universitário e, assim, experimentam essas situações de ansiedade de forma solitária. É imprescindível que as escolas médicas ampliem a oferta de grupos de apoio para acolher essas situações, de modo a promover a saúde mental dos discentes. Além disso, é fundamental que ações desse tipo sejam garantidas na grade curricular a fim de facilitar a participação de todos.

O Redemed $^{\odot}$ apresenta uma abordagem de gerenciamento do estresse baseada no desempenho da meditação e inclui atividades de relacionamento interpessoal. Foi referido como um dos grupos nos quais os entrevistados que receberam a intervenção se sentiram mais acolhidos (da mesma forma que se sentiam em relação às famílias e aos centros acadêmicos). Portanto, vale ressaltar que cursos como esse podem ser um programa interessante para esse fim.

Por fim, quanto à diferença estatística observada em relação à maior participação do sexo feminino (64\%) no grupo intervenção em comparação ao grupo controle $(42,5 \%)$, considerou-se um viés de seleção, uma vez que a aderência ocorreu de acordo com o interesse dos participantes em participar do programa Redemed ${ }^{\circ}$. Porém, quando foi realizada regressão linear normal múltipla, para corrigir o efeito de confusão de gênero, apesar da diferença entre os grupos, esse resultado não impactou a intervenção na evolução do estresse (Tabela 2). Os voluntários que formaram o grupo controle eram estudantes que não tinham interesse ou tempo para participar das reuniões do programa e que foram convidados pelos estudantes que queriam fazer o programa. No entanto, o fato de as mulheres terem apresentado maior adesão ao Redemed $^{\oplus}$ pode estar relacionado ao maior interesse delas em buscar medicinas complementares, conforme demonstrado por Kristoffersen et al. $^{29}$ em seu estudo, que incluiu 12.982 indivíduos com diferença de gênero na prevalência de uso de medicinas complementares.

Como limitação do estudo, destacamos a não randomização dos grupos controle e intervenção, e um acompanhamento maior do grupo intervenção, como um grupo de apoio para que eles pudessem praticar a meditação juntos mais vezes por semana. Além idsso, houve perdas no grupo intervenção e principalmente no controle, com respostas incompletas dos questionários. Talvez a aplicação de questionários on-line com obrigatoriedade de cada item possa ajudar em estudos futuros.

No Brasil, o número de publicações sobre meditação indica que a produção na área ainda é restrita e recente ${ }^{14}$. Um desafio para esse campo de pesquisa é tornar as pessoas em geral comprometidas com a prática da meditação. Investigações qualitativas e quantitativas sobre como as pessoas experimentam essa prática, os efeitos dela, as dificuldades encontradas, o que as motiva a procurá-la e mantê-la poderiam ajudar a entender estratégias para a aplicação da meditação na vida cotidiana e nos diferentes momentos da formação de um médico.

Este estudo mostrou resultados positivos na redução dos sintomas percebidos de estresse por estudantes de Medicina com o uso de um programa de oito semanas que utilizava a meditação como técnica central. A meditação, de maneira semelhante a qualquer estratégia comportamental, requer motivação, persistência e disciplina. Embora não seja um mecanismo em si, sabe-se que a prática e a repetição desempenham um forte papel mediador no efeito da meditação no estresse ${ }^{30}$. Os grupos de apoio podem colaborar com a motivação, que talvez seja o aspecto mais importante para o início da prática, bem como para sua manutenção e regularidade ${ }^{14}$.

\section{CONCLUSÃO}

Este estudo mostrou que o programa Redemed ${ }^{\odot}$ foi eficaz na redução do estresse percebido dos estudantes de Medicina que participaram dessa intervenção quando comparados ao grupo controle $(\mathrm{p}=0,000)$.

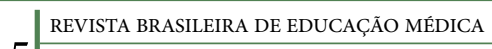

5 44 (3) : e104; 2020 
Assim, sugere-se que esse programa, que combina práticas de meditação e atividades em grupo, possa ser uma ferramenta interessante a ser implementada nas escolas de medicina com o objetivo de gerenciar o estresse de futuros profissionais. É importante que as faculdades de Medicina abram espaços para acolher e ouvir os alunos em relação aos diferentes tipos de sofrimento que eles podem enfrentar durante o curso.

\section{REFERÊNCIAS}

1. Fiorotti KP, Rossoni RR, Borges LH, Miranda AE. Transtornos mentais comuns entre os estudantes do curso de medicina: prevalência e fatores associados. J Bras Psiquiatr. 2010;59 (1):17-23.

2. Dyrbye LN, Thomas MR, Shanafelt TD. Systematic review of depression, anxiety, and other indicators of psychological distress among U.S. and Canadian medical students. Acad Med. 2006;81:354-73.

3. Costa EFO, Santos SA, Santos ATRA, Melo EV, Andrade TM. Burnout syndrome and associated factors among medical students: a cross-sectional study. Clinics. 2012; 67:573-80.

4. Alves TCTF. Depressão e ansiedade entre estudantes da área de saúde. Rev Med (São Paulo). 2014;93(3):101-5.

5. Brenneisen MF, Souza SI, Silveira PS, Itaqui LHM, de Souza AR, Campos EP, et al. Factors associated to depression and anxiety in medical students: a multicenter study. BMC Med Educ. 2016;16(1):282

6. Meyer C, Guimarães ACA, Machado Z, Parcias SR. Qualidade de vida e estresse ocupacional em estudantes de medicina. Rev Bras Educ Med. 2012;36(4):489-98.

7. Fares J, Al Tabosh H, Saadeddin Z, El Mouhayyar C, Aridi H. Stress, burnout and coping strategies in preclinical medical students. N Am J Med Sci. 2016;8(2):75-81.

8. Lemos KM, Neves NMBC, Kuwano AY, Tedesqui G, Bitencourt AGV, Neves FBCS, et al. Uso de substâncias psicoativas entre estudantes de Medicina de Salvador (BA). Rev Psiq Clín. 2007;34(3):118-24.

9. Florence KC, Andrade AG, Bassit AZ, Vilella NM, Boccuto F. Uso de álcool e drogas por estudantes de medicina da Unesp. Rev Bras Psiquiatr. 1999;21(2):95-100.

10. Dobkin PL, Hutchinson TA. Teaching mindfulness in medical school: where are we now and where are we going? Med Educ. 2013;47(8):768-79.

11. Menezes CB, Dell'Aglio DD. Por que meditar? A experiência subjetiva da prática de meditação. Psicol Estud. 2009;14(3):565-73.

12. Tesser CD, Luz MT. Medical rationalities and integrality. Ciênc Saúde Colet. 2008;13(1):195-206.

13. Carneiro DM. Ayurveda: saúde e longevidade na tradição milenar da Índia. São Paulo: Pensamento; 2009.

14. Menezes CB, Dell'Aglio DD, Bizarro L. Meditação, bem-estar e a ciência psicológica: revisão de estudos empíricos. Interação Psicol. 2011;15(2):239-48.

15. Kozasa EH. A prática de meditação aplicada ao contexto da saúde. Saúde Colet. 2006;3(10):63-6.

16. Shapiro SL, Shapiro DE, Schwartz GE. Stress management in medical education: a review of the literature. Acad Med. 2000;75(7):748-59.

17. Andrews S. O stress a seu favor. São Paulo: Agora; 2003.

18. Luft CB, Sanches SO, Mazo GZ, Andrade A. Brazilian version of the Perceived Stress Scale: translation and validation for the elderly. Rev
Saúde Pública. 2007;41:606-15.

19. Reis RS, Hino AA, Anez CR. Perceived stress scale: reliability and validity study in Brazil. J Health Psychol. 2010;15:107-14.

20. Hewitt PL, Flett GL, Mosher SW. The perceived stress scale: factor structure and relation to depression symptoms in a psychiatric sample. J Psychopathol Behav Assess. 1992;14(2): 247-57.

21. Mimura C, Griffths P. A Japanese version of the perceived stress scale: translation and preliminary test. Int J Nurs Stud. 2004;4(4):379-85.

22. Remor E. Psychometric properties of a European Spanish Version of the Perceived Stress Scale (PSS). Span J Psychol. 2006;9(1):86-93.

23. Kuhlmann SM, Huss M, Burger A, Hammerle F. Coping with stress in medical students: results of a randomized controlled trial using a mindfulness-based stress prevention training (MediMind) in Germany. BMC Med Educ. 2016;28(16):316.

24. Regehr C, Glancy D, Pitts A, LeBlanc VR. Interventions to reduce the consequences of stress in physicians: a review and meta-analysis. J Nerv Ment Dis. 2014;202(5):353-9.

25. McConville J, Mcaleer R, Hahne A. Mindfulness training for health profession students - the effect of mindfulness training on psychological well-being, learningand clinical performance of health professional students: a systematic review of randomized and nonrandomized controlled trials. Explor (N. Y.). 2017;13(1):26-45.

26. Van Dijk I, Lucassen PL, Akkermans RP, Van Engelen BG, Van Weel C, Speckens AE. Effects of mindfulness-based stress reduction on the mental health of clinical clerkship students: a cluster-randomized controlled trial. Acad Med. 2017;92(7):1012-21.

27. Ngô TL. Revuedes effets de la méditation de pleine conscience sur la santé mental e et physique et surses mécanismesd’action. Santé Ment Qué. 2013;38(2):19-34.

28. Moreira SNT, Vasconcellos RLSS, Heath N. Estresse na formação médica: como lidar com essa realidade? Rev Bras Educ Med. 2015;39(4):558-64

29. Kristoffersen AE, Stub T, Salamonsen A, Musial F, Hamberg $\mathrm{K}$. Gender differences in prevalence and associations for use of CAM in a large population study. BMC Complement Altern Med 2014;14(463):1-9.

30. Menezes CB, Dell'Aglio DD. The relationship between the practice of sitting and silent meditation, and psy-chological well-being, and the effects of personality traits. Am J Psychol. 2010;44:1-10.

\section{CONTRIBUIÇÃO DOS AUTORES}

Madeline Susan Andrews, Emmanuel A. Burdmann e Karina Pavão Patrício fizeram contribuições substanciais para a concepção e o desenho do artigo. A coleta, análise e interpretação dos dados foram etapas que tiveram a participação de Fernanda Martin Catarucci, Talita Cardoso Rossi, Ivan da Silva Beteto, Vânia Hercília Talarico Bruno, Pedro Henrique Leonetti Habimorad e Karina Pavão Patricio. Após essa primeira etapa, os pesquisadores Fernanda Martin Catarucci, Talita Cardoso Rossi, Ivan da Silva Beteto, Vânia Hercília Talarico Bruno e Pedro Henrique Leonetti Habimorad elaboraram o artigo. Por fim, houve uma importante revisão crítica do conteúdo intelectual realizda por Madeline Susan Andrews, Emmanuel A. Burdmann e Karina Pavão Patrício. 


\section{CONFLITO DE INTERESSES}

Os autores declaram não haver conflitos de interesse neste estudo.

\section{ENDEREÇO PARA CORRESPONDÊNCIA}

Fernanda Martin Catarucci. Departamento de Saúde Pública da Faculdade de Medicina de Botucatu da Universidade Estadual Paulista "Júlio de Mesquita Filho" (FMB/Unesp), Avenida Professor Mário Rubens Guimarães Montenegro, s/n, Botucatu, SP, Brasil. CEP: 18.618-687.

E-mail: fernandacatarucci@gmail.com 